

\section{ÍNDICE}

\section{ÁMBITOS PERSONALES PERSONAL ÁMBITOS}

Crisis de Venezuela: Análisis y perspectivas según los titulares de la prensa argentina, española y china

Venezuela's crisis: Analysis and perspectives in the headlines of Argentine, Spain and China press

Hui Feng Liu

Tratamiento informativo de la violencia de género: asesinatos de mujeres. Análisis de la agencia EFE

Informative treatment of gender violence: murders of women. Analysis of the press agency EFE Rosa Rodríguez Cárcela, Agustín López Vivas

MONOGRAFICOS MONOGRAPHS

Presentación Monográfico. El universo transmedia de los medios de comunicación universitarios: acción dentro y fuera del aula en la sociedad postdigital

Antonia Isabel Nogales-Bocio, Ángels Álvarez villa

El papel transformador de la radio universitaria en materias teóricas ajenas a la comunicación

The transformative role of college radio in theoretical subjects outside communication

Miguel Ángel Díaz Monsalvo

La radiodifusión universitaria: acción discursiva radiofónica para la divulgación de la ciencia University Radio Broadcasting: Radiophonic Discursive Action for the Science Popularization Jorge Sadi Durón, Joel Zapata Salazar

El uso corporativo de Instagram en las universidades privadas españolas. Estudio comparativo de treinta y cinco universidades

The corporate use of Instagram in spanish private universities. Comparative analysis of thirty-five private universities 
La radio universitaria como herramienta de inclusión social: OndaCampus en contextos como la cárcel y barrios desfavorecidos

The university radio as a tool for social inclusion: OndaCampus in contexts like the prison and disadvantaged neighborhoods

Leonor Real Adame, Daniel Martín-Pena, Macarena Parejo Cuéllar

Hacer radio universitaria en la era de YouTube: uso de la plataforma de vídeos a demanda en el contexto mexicano

Make college radio in the age YouTube: use of video on demand platform in the Mexican context Marina Vázquez Guerrero

\section{ARTÍCULOS ARTICLES}

O impresso e o digital nos modelos de negócios de jornais locais: uma análise do Sermos Galiza

The press and digital in business models from local media: Analysis of newspaper Sermos Galiza

Giovanni Ramos

Publicidad y cáncer en la prensa escrita (1903-1912)

Advertising and cancer in the written press (1903-1912)

Laura Almudéver-Campo, Ramón Camaño-Puig

Game rules vs. fandom. How Nintendo's Animal Crossing fan-made content negotiates the videogame meanings

Las reglas del juego vs. el fandom. Cómo el contenido hecho por fans de Nintendo Animal Crossing negocia los significados del videojuego

Jose A. Moreno

La comedia de situación y su análisis textual: evolución de los elementos constructivos del formato

The sitcom and its textual analysis: evolution of the constructive elements of the format

Darío Martín Sánchez

Análisis del discurso emocional de Donald Trump en la campaña electoral de $\mathbf{2 0 1 6}$ Analysis of Donald Trump's emotional speech on the 2016 election campaign 
Innovar, comunicar y transformar (en) la Universidad

Innovate, communicate and transform (at) the University

María Sofía Bernat

288-292

Transparencia en los medios: Un requisito imprescindible para medir la rentabilidad social en radio y televisión

Transparency in the media: An essential requirement to measuresocial profitability in radio and television

Amanda Salazar

Los estudios feministas en comunicación: representación de las mujeres en la revolución tecnológica

Feminist studies in communication: representation of women in the technological revolution 


\title{
La radio universitaria como herramienta de inclusión social: OndaCampus en contextos como la cárcel y barrios desfavorecidos
}

\author{
The university radio as a tool for social inclusion: OndaCampus \\ in contexts like the prison and disadvantaged neighborhoods
}

\section{Leonor Real Adame, Universidad Extremadura,}

Plazuela Ibn Marwan, s/n, 06001 Badajoz

radio@ondacampus.es | Orcid: orcid.org/0000-0002-1123-5919

Daniel Martín-Pena, Universidad Extremadura,

Plazuela Ibn Marwan, s/n, 06071 Badajoz

danielmartin@unex.es | Orcid: orcid.org/0000-0003-2676-5821

Macarena Parejo Cuéllar, Universidad Extremadura,

Plazuela Ibn Marwan, s/n, 06071 Badajoz

macarenapc@unex.es | Orcid: orcid.org/0000-0002-5292-2731

DOI: http://dx.doi.org/10.12795/Ambitos.2020.i47.07

\section{Resumen}

La evolución de las radios universitarias en España ha conseguido que su carácter formativo se amplíe hasta alcanzar su función social. Si bien las emisoras que pertenecen a las universidades cumplen la función esencial de prestar una formación adicional y práctica al alumnado, también han permitido conectar, de manera real, la 
institución académica con la ciudadanía. De este modo, surgen iniciativas en las emisoras, en colaboración con algunos servicios universitarios y asociaciones externas a la propia institución docente y científica, que promueven la cultura, la difusión del conocimiento $\mathrm{y}$, como principal factor, la inclusión social de las personas que participan en los mismos. A lo largo de este trabajo, hablamos y analizamos dos iniciativas de carácter social: "La Ventana de la Ciencia", programa de radio dedicado a la divulgación científica, realizado íntegramente por internos del Centro Penitenciario de Badajoz y el proyecto "E-Misión Color", cuyo objetivo consiste en empoderar, en materia de comunicación, a los ciudadanos de los barrios más desfavorecidos de la margen de la ciudad de Badajoz. A partir de una recolección de datos y del uso de una metodología cualitativa basada en la entrevista, este estudio permite demostrar las funciones naturales, accidentales y transversales de la radio universitaria. Los datos obtenidos a partir de este trabajo corroboran, en este sentido, el valor de la radio universitaria como herramienta de reinserción y resocialización.

\section{Abstract}

The evolution of university radios in Spain has achieved that its formative character is extended to reach its social function. Although the stations belonging to the universities fulfill the essential function of providing additional and practical training to students, they have also made it possible to connect, in a real way, the academic institution with the citizens. In this way, initiatives arise in the stations, in collaboration with some university services and associations outside the teaching and scientific institution itself, which promote culture, the dissemination of knowledge and, as the main factor, the social inclusion of the people who participate in them. Throughout this work, we talk and analyze two initiatives of a social nature: "The window of science", a radio program dedicated to scientific dissemination, carried out entirely by inmates of the Badajoz Penitentiary Center and the project "E-Mission Color", Whose objective is to empower, in matters of communication, the citizens of the most disadvantaged neighborhoods on the banks of the city of Badajoz. From a data collection and the use of a qualitative methodology based on the interview, this study allows to demonstrate the natural, accidental and transversal functions of the university radio. The data obtained from this work corroborate, in this sense, the value of university radio as a tool for reintegration and resocialization.

Palabras clave: Comunicación, radio, universidad, sociedad, inclusión Keywords: Communication, radio, university, society, inclusion

\section{INTRODUCCIÓN}

Las radios universitarias han dado claras muestras de su función social como ente integrador y democrático. El objetivo que se persigue en este trabajo es dar a conocer, 
por un lado, y analizar, por otro, dos experiencias puestas en marcha desde la radiotelevisión de la Universidad de Extremadura, OndaCampus. Se trata de los proyectos "La ventana de la ciencia" y "E-Misión Color". A través de ellos, durante dos años se ha trabajado a través de la radio con personas en riesgo de exclusión social. Como hipótesis fundamental, desde los inicios de la puesta en marcha de estas actividades, se partía de la clara convicción, de que la radio universitaria supone una herramienta pedagógica, social, formativa y terapéutica de alto valor. La experiencia vivida ha permitido perfilar un proceso investigativo consistente en la confección de entrevistas a los diferentes agentes implicados en la acción. De la selección de palabras repetidas como relevantes, a partir de un análisis etiquetador morfosintáctico, ha sido posible concluir una serie de funciones sociales fundamentales naturales, accidentales y transversales inherentes al medio universitario estudiado.

\section{LA FUNCIÓN SOCIAL DE LOS MEDIOS DE COMUNICACIÓN}

Los medios de comunicación están en permanente contacto con la sociedad. Estos obtienen de manera natural, una serie de funciones sociales, que instauran un compromiso con la sociedad. Bretones (1997) describe cuatro líneas emergentes y constantes en las investigaciones sobre los medios de comunicación de masas y la asignación de las funciones sociales que se les atribuye:

1) Función manipuladora de los medios: relacionada directamente con la teoría hipodérmica de Wolf (1991). La misma asigna a los medios una capacidad para influir y manipular a los individuos. El mensaje penetra en ellos de forma similar al que lo hace una bala que impacta sobre el cuerpo físico al que penetra (Muñoz et al., 1992).

2) Función movilizadora: establece una relación de acomodación entre los mensajes que emiten los medios y las motivaciones que orientan la conducta (Mora, 2016).

En la década de 1940, comienzan a aparecer estudios donde se relativizan los efectos manipuladores de los medios, llegando a alcanzar la idea de que no se les puede atribuir este efecto directo. Son ejemplo, las situaciones de pánico generadas tras las emisiones del famoso programa de radio "La Guerra de los mundos" de Orson Welles. La composición de los mensajes del programa estaba representada de forma verídica, lo que causó un gran impacto en los oyentes, como si lo que hubieran escuchado, perteneciera a una catástrofe real.

Por otro lado, el modelo clásico de Lasswell, de 1948, supone una teoría enmarcada en el paradigma funcionalista: "Quién dice qué, en qué canal, a quién y con qué efecto". Esta secuencia de preguntas se resume en dos grandes bloques: 1) los 
centros de atención en la investigación sobre los efectos de los mensajes de los medios, el análisis de los profesionales, del contenido del mensaje, del tipo de medio, el público y de los efectos; y 2) los distintos frentes a los que debe encontrarse la causa o causas de los efectos de los mensajes de los medios (Mora, 2016).

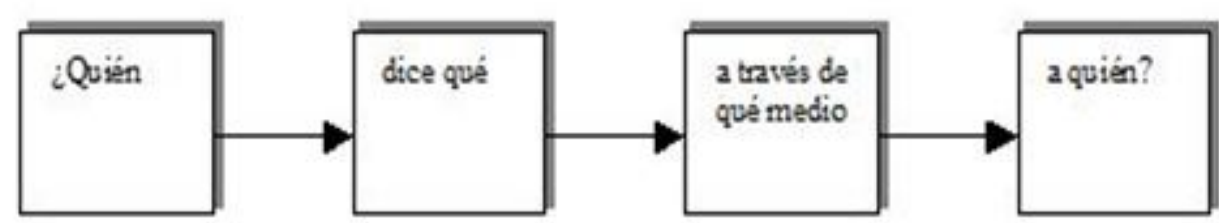

\section{¿En qué circunstancias?}

\section{¿Con qué propósito?}

\section{¿Con qué efecto?}

Figura 1: Perfeccionamiento del modelo de Lasswell hecho por Bradok (1958) Fuente: McQuail y Windhal (1997, p. 42).

3) Función de control social: como el resultado de un ejercicio de poder del que forman parte los medios como eslabón final. Estos escenifican los recursos de un grupo de poder y su función radica en producir socialmente la escenificación del poder al tiempo que escenifican el "control como resultado". Si ese control se define desde un punto de vista social, se interpreta a los medios como formando parte de este proceso, desempeñando en él una función relevante de control no obligatoriamente dirigido desde una élite de poder. Es decir, la función de control social sería una gestión de la opinión pública (Bretones, 1997).

4) Función de reproducción cultural: gira en torno a los papeles "reproductivos" que los medios desempeñan en la "construcción" de significados (en forma de mensajes), y por medio de una "socialización indirecta" de la sociedad en los "símbolos" comunes (Mora, 2016).

\subsection{La función social en la universidad: la radio universitaria como medio de comunicación alternativo}

La universidad tiene un permanente contacto con la sociedad. Por este motivo, no puede abandonar su Responsabilidad Social Universitaria. La institución tiene como principal función social la educación superior. Sin embargo, en su evolución no puede dejar a un lado a la ciudadanía. Como bien es sabido las funciones tradicionales de la Universidad son tres: la docencia o la formación; la investigación y, finalmente, la 
transferencia del conocimiento. Sin embargo, Parejo, Vivas y Martín-Pena (2018, p.2025) entienden que puede haber una universidad alternativa. Apuntan algunas ideas sobre lo que "la Universidad tiene que ser además", más allá de las funciones tradicionales anteriormente mencionadas y siguiendo postulados de Bueno Campos (2017), Ferrer (2014) y Sols (2016):

1. La función crítica y social como objetivo, en defensa de la libertad y la igualdad de los ciudadanos (Sols, 2016).

2. La cultura humanista y el pensamiento complejo como instrumento. Como apuntan los autores al sistema educativo universitario le compete formar estudiantes con una gran sensibilidad social.

3. La utilización de la palabra como método, una palabra alejada de populismo y demagogia, "sino la palabra precisa y ajustada a la realidad, por un lado, y metódica y ordenada por otro, que describe y trata de mover la conciencia (...) $\mathrm{Ni}$ que decir tiene, la magnitud que tienen, por ejemplo, las radios universitarias como portavoces de la palabra universitaria y animadoras de la divulgación científica".

4. El debate y el enfrentamiento como actitud, "si la universidad tiene por objeto la función social de transformar la realidad, el talante no puede ser de resignación, docilidad o conformidad".

5. La formación y el aprendizaje como marco de referencia. Aquí, la divulgación científica se comporta como una herramienta eficaz de cambio social.

6. La Universidad debe ambicionar la utopía como fin. "No se trata de defender quimeras o de ambicionar ilusiones y fantasías". Los autores apuntan resistencias y dificultades en este discurrir:

- Dificultades de carácter económico, proveniente de los pocos recursos de que se disponen.

- Impedimentos de orden social, proveniente también desde el interior de la propia universidad.

Sin embargo, a pesar de todas estas dificultades la Universidad debe buscar institucionalmente la utopía, consistente en:

- Estar convencidos de la importancia que la Universidad presenta como herramienta social. Naturalmente, desde un punto de vista científicotécnico, y en consecuencia económico, pero también desde una perspectiva social. 
- Seleccionar bien a los profesores. La institución necesita de docentes comprometidos con su acción científica y social. Y para ello, es esencial que no se incurra en el relativismo del currículum porque sí. El sistema competitivo está derivando en grupos de intereses, redes clientelares y ausencia de compromiso ético.

- Buscar la proyección social, para que la universidad, a través de la cultura y la producción intelectual beneficie al conjunto de la sociedad, fundamentalmente a los más desfavorecidos.

- Constituirse en conciencia de la sociedad, en la búsqueda de la igualdad, la justicia y la libertad.

Es en este punto donde las emisoras universitarias pueden ayudar a contribuir en la responsabilidad social universitaria. Tienen la misión de aunar esfuerzos con los diferentes servicios, estableciendo contacto directo con la sociedad a través de sus acciones. De esta manera, se rompen las barreras del campus universitario con el resto de la sociedad, estableciendo un puente sonoro entre ambas.

\subsubsection{La función social en las emisoras universitarias}

Las emisoras universitarias viven de cara a la sociedad. Desde sus inicios esta ha sido una premisa irrenunciable. El detonante de la radiodifusión universitaria de cariz social surgió en América Latina como consecuencia de la denominada Reforma de Córdoba de 1918 en Argentina, que dotaría a la universidad de algo de modernidad (PintoZúñiga y Martín-Pena, 2017).

Fue en junio de ese año cuando los jóvenes universitarios cordobeses iniciaron unas movilizaciones en favor de la democratización de la enseñanza, a las que se adherirán, poco a poco, todas las academias del entorno (Aguaded y Martín-Pena, 2013).

Las reivindicaciones reformistas abogaban por la renovación de las estructuras y los objetivos de las universidades, la implementación de nuevas metodologías de estudio y enseñanza, el razonamiento científico frente al dogmatismo, la libre expresión del pensamiento, el compromiso con la realidad social y la participación del claustro estudiantil en el gobierno universitario (Rotman, 2014). Pero, lo que resultó trascendental para las futuras radios universitarias era uno de los principios por los que luchaba esta reforma: la extensión universitaria y el compromiso con la sociedad, en busca de un fortalecimiento de la función social de la universidad, y la proyección al ciudadano de la cultura universitaria y la preocupación por los problemas nacionales. Una verdadera incorporación de la difusión cultural y la extensión universitaria como tareas propias de las instituciones universitarias (Pinto-Zúñiga y Martín-Pena, 2017). 
Para acercarnos a la realidad social de la radio universitaria en España, debemos aproximarnos a la radio educativa (Martín-Pena y Aguaded, 2016). La radio como recurso educativo llega a España mucho más tarde que en América Latina, Estados Unidos o Centroeuropa, aunque el uso de la radio en la Guerra Civil Española se estudia como ejemplo de utilización del medio para informar, adoctrinar y ensayar el uso que después se le daría en la II Guerra Mundial (Marta y Segura, 2011).

"Desde sus primeros momentos de vida, la radio fue vinculada a proyectos educativos, no solo en España sino en otros países europeos y americanos. Se convirtió, siempre que la censura se lo permitió, en un medio prestigioso desde el punto de vista informativo y, en tiempos de guerra, supo mostrar sus cualidades como instrumento para el adiestramiento ideológico al servicio de los poderes beligerantes" (González Conde, 2003, p.2).

La radio educativa forma parte de los cimientos de la universidad, pero hoy en día, en la mayoría de los casos, las estaciones universitarias, salvo el caso de Radio UNED, no persigue el objetivo de ser una emisora educativa.

A lo largo de su trayectoria histórica, esta radio adquiere diferentes modalidades: instructiva, comunitaria, popular o formativa, pero siempre mantiene dos características que la identifican: por un lado, su carácter no comercial y, por otro, su finalidad social (Marta y Segura, 2011). Atendiendo a esta perspectiva, González Conde (2003, p.11) apunta los siguientes planos de actuación que ha tenido la radio educativa:

a) Como apoyo directo a intereses y movimientos sociales determinados. En los años sesenta, la radio se convierte en foro de expresión de ideas revolucionarias. Las luchas sociales de aquellos años se desarrollan vinculadas a proyectos de radios educativas.

b) Como colaboradora no solo en la extensión de la instrucción educativa formal para amplísimas comunidades sino, y de forma más general, como impulsora para el cambio y desarrollo social, de acuerdo a programas sociales promovidos por la UNESCO y otros organismos internacionales, para los países menos desarrollados.

c) Y, por último, como instrumento para la educación no formal, a través de la participación ciudadana, en las radios populares y comunitarias.

En lo referente a las emisoras universitarias, nos encontramos, en la mayoría de los casos, ante un modelo de emisora que comparte la definición de Merayo (2000) aludiendo a que no persigue objetivos comerciales. Por tanto, no se trata tanto de formar al oyente, sino de divulgar el conocimiento científico a la 
vez de establecer una conexión entre la universidad y la sociedad (ReginoPinto y Martín-Pena, 2017).

En este punto, la puesta en marcha de emisoras universitarias en España, como apuntan Martín-Pena y Espino (2014, p.18), "suele tener como fin, en la mayoría de los casos, el abrir un canal de participación, donde se expresen inquietudes o se visualice el día a día de la institución, siempre extendiendo a la sociedad la cultura, la ciencia o el conocimiento general que la propia institución genera.

Según Marta y Martín-Pena (2014), es en la segunda fase del desarrollo histórico de las radios universitarias españolas, cuando se produce una evolución en la mentalidad de las mismas. A partir del caso de la aparición en 2007 de UniRadio Huelva. Se señala a esta estación universitaria como un caso significativo en lo que a responsabilidad social se refiere. La estación onubense se convierte en la primera emisora de radio universitaria que emerge en Andalucía y que logra una concesión de dicha comunidad autónoma para operar como emisora cultural por medio de una asociación cultural. Es la primera emisora que realiza publicidad social y propone una programación que busca un acercamiento progresivo a la sociedad, asunto que se torna en factor clave del apogeo vivido por las emisoras universitarias en esos años, y que hará que muchas otras emisoras como OndaCampus o 98.3 Radio imiten parte de sus iniciativas (Pinto-Zúñiga y Martín-Pena, 2017).

La autorización administrativa lograda por UniRadio vino condicionada por la exigencia de una programación compuesta fundamentalmente de contenidos de carácter divulgativo, cultural, educativo y solidario. Contreras (2012) indica que la acción y la intervención social son parte importante de la parrilla de la emisora, para lo cual cuenta con la colaboración de colectivos que provienen tanto de adentro como de afuera de la comunidad universitaria. Como señalan Contreras, Mairena y Aguaded (2014, p.125) desde el inicio se ha seguido una línea de trabajo en la que se construye una parrilla de programación de cariz social, bajo el nombre de Propósito Penélope que "se fundamenta en la responsabilidad de tejer una red de colaboración, solidaridad y trabajo colectivo en pos de lograr una sociedad más avanzada", toda una declaración de intenciones que convierte a esta estación universitaria en el paradigma del perfil social y de servicio público de las radios universitarias españolas.

Así, tal y como apunta López (2005), estamos ante un nuevo reto para la universidad, donde se sustituye el concepto de proyección social, instaurándose el de responsabilidad social universitaria. Casas (2005) subraya la importancia de la universidad para lograr una transformación de la sociedad, así como el progreso de la misma. Antequera y Obregón (2002) hablan del papel que tiene la radio en los procesos de desarrollo social y cultural, lo que lleva a la utilización de este medio como una herramienta de cambio, que promueva la participación y el protagonismo de 
los oyentes, y les permita ser los responsables de dicha transformación. Al respecto de la transferencia social y del conocimiento, hay que destacar la función de servicio público que cumplen las emisoras universitarias, formando, informando e integrando al oyente como parte importante del medio (Aguaded y Contreras, 2011). Se consigue de esta forma que una persona perteneciente al tejido social "sienta como suyo un lugar que siempre se ha visto como reservado para las élites del conocimiento" (Contreras, 2012, p.153).

En 2011, Marta y Segura nos muestran el avance de las tecnologías en las radios universitarias, apunte que puede ser aprovechado para enlazar como posible consecuencia y herramienta para estrechar aún más los lazos entre la universidad y la sociedad. En este sentido, para Garcés (2007), "los finales del siglo XX e inicios del $\mathrm{XXI}$ atestiguaron también un proceso acelerado de renovación tecnológica que, en el caso específico de la radio, implicó la digitalización de la producción y las trasmisiones, junto a un crecimiento exponencial del número de páginas web, tanto de emisoras de alcance nacional como regional". En cuanto a las nuevas opciones y alternativas que plantea la radio online, López Vidales (2011, p.24) hace referencia al término 'radiovisión' y expone que "pone al alcance del cibernauta hiperenlaces, datos, podcasting, ciberrevistas, juegos, esquemas, gráficos, etc. Mientras, se dispone de acceso a servicios como las compras online, selección de música, intervención en foros, buscadores temáticos, etc". Un contenido transmedia utilizado en los nuevos formatos $y$, especialmente fuertes en las emisoras universitarias, que crean vínculos extraordinarios con la sociedad y que estas mismas radios aprovechan para la proliferación de proyectos en diferentes contextos sociales.

La radio universitaria se convierte en el cauce privilegiado de expresión de las inquietudes de la comunidad (González y Ramos, 2012). Para López (2005), la radio es el mejor medio técnico interactivo para actuar en el entorno, ya que permite que se establezca un contacto directo entre el comunicador y la sociedad, y señala que el comunicador es la propia universidad, el que se encarga de informar y aportar cultura además de difundir el saber que se genera en la misma. Al respecto, Casajús (2011) señala que las radios enmarcadas en el contexto universitario, y que funcionan con el respaldo de las universidades que las albergan, son parte del sistema de medios universitarios y se insertan en la sociedad, por lo que también conforman el sistema de medios de comunicación social. Esto da lugar a una auténtica "red de activos sociales de su entorno inmediato que encuentran un espacio de participación, enmendando los errores de comunicación del sistema comercial" (Contreras, Mairena y Aguaded, 2014, p.128).

"Dar voz a la sociedad que rodea a estas estaciones, así como impulsar y colaborar con las causas sociales, se ha convertido en parte del día a día de estas emisoras, que constituyen el lugar idóneo para ello, ya que no existen presiones comerciales o 
partidistas que limiten la libertad de expresión de los colaboradores" (Pinto-Zúñiga y Martín-Pena, 2017, p.173).

\section{RADIO UNIVERSITARIA COMO HERRAMIENTA DE INCLUSIÓN SOCIAL, EL CASO DE ONDACAMPUS}

Bajo esta premisa y, en el contexto de esta nueva etapa de la radio universitaria, claramente marcada por el carácter social que debe desempeñar, OndaCampus, la radio de la Universidad de Extremadura, se ha abierto activamente a la sociedad, albergando en la actualidad más de 30 programas realizados por alumnado de la UEx, instituciones, asociaciones y por la sociedad, en general.

Su claro carácter social se refleja también en el desarrollo de los talleres de radio en los que los alumnos de la UEx se ven apoyados nuevamente por la estación de la universidad en el carácter práctico, así como en la puesta en marcha de dos proyectos clave para la justificación de este estudio: el proyecto de divulgación científica en la cárcel de Badajoz "La Ventana de la Ciencia" y el programa de radio en un barrio desfavorecido de la misma ciudad denominado "E-Misión Color".

\section{1. "La Ventana de la Ciencia"}

En "La Ventana de la Ciencia" es la propia población reclusa del Centro Penitenciario de Badajoz la que tiene una función proactiva en la confección de los programas de radio. La idea que se persigue es que estos espacios, dedicados a la divulgación de la ciencia, sean realizados íntegramente por personas que en estos momentos se encuentran en situación de privación de su libertad. Por ello, son los propios presos, una docena de personas, quienes abordan diversos temas de investigación de interés general.

Ellos son los encargados de construir un discurso en torno a la ciencia y la tecnología (temas de divulgación, resultados de investigaciones de científicos de su entorno...) para el cual no solo deben ponerse de frente a los micrófonos sino que previamente tienen que desarrollar labores de documentación, producción y análisis de tipo colaborativo para la construcción de un mensaje con ciertas particularidades al haber sido realizado desde una mirada diferente, la del encierro.

Resocialización y reeducación son, por tanto, las piedras angulares de esta actividad que utiliza la ciencia y el medio radio como los canales para la consecución de los propósitos previstos.

De alguna manera, producir los contenidos de este programa es ya algo más que motivador para los conductores de este espacio, ya que pueden volver a establecer un 
diálogo a través de la ciencia con la sociedad. Además, no debe perderse de vista que no existía ningún espacio de este tipo en España. "La Ventana de la Ciencia" es el primer programa de ciencia y tecnología realizado desde la cárcel.

En este sentido, el espacio destaca también por su cariz colaborativo y construido a partir de esta metodología de trabajo que favorece la reinserción social.

\subsection{E-Misión Color}

"E- Misión Color" es una herramienta modulada como altavoz social, una plataforma de comunicación gestada para calibrar, mostrar, documentar, retratar y absorber la esencia de los barrios de la ciudad. Un medio de comunicación al que ponerle rostro y voz, un objetivo social para plasmar la realidad de las zonas urbanas que conforman el núcleo metropolitano.

Imagen, testimonio y sonido como ingredientes comunicativos. Una receta documental que compone el cuaderno de bitácora de "E- Misión Color".

Con "E- Misión Color" desde OndaCampus se hace referencia a las nuevas tecnologías a través de la "E". Seguimos con nuestra misión, de colorear los barrios que todos creemos en blanco y negro.

\section{METODOLOGÍA}

OndaCampus es la radio televisión de la Universidad de Extremadura. Y, precisamente, uno de los objetivos y funciones principales de una radio universitaria, es su función social y servicio a la sociedad. Servir de medio de comunicación a los que no tienen voz en los medios convencionales. Su principal propósito, por tanto, es crear un medio de comunicación social al servicio de la comunidad. Un medio abierto que sirva para la expresión de toda persona que quiera expresarse. Además, promover la difusión de conocimientos y acercar los medios de comunicación a todos los colectivos sociales. Fomentar y despertar vocaciones comunicativas en los usuarios y las usuarias.

De ahí que el desarrollo y ejecución de estos dos proyectos comentados en el apartado anterior (3.1 y 3.2), hayan permitido acercar el medio y trabajar con personas en riesgo de exclusión como son los presos de la cárcel y los vecinos del barrio del Gurugú, en Badajoz. Pero además, también haya brindado la oportunidad de analizar, desde un punto de vista, investigativo, la capacidad, o el papel, que juega OndaCampus en el proceso de la inclusión social. Este término es definido por la UE como aquel que asegura que aquellas personas que están en riesgo de pobreza y exclusión social, tengan las oportunidades y recursos necesarios para participar completamente en la vida económica, social y cultural disfrutando un nivel de vida y bienestar que se considere normal en la sociedad en la que ellos viven. Este término 
pone énfasis, en este sentido, en el derecho de las personas de "tener una vida asociada siendo un miembro de una comunidad.

Precisamente, el objetivo de este estudio se ha basado en medir desde una perspectiva cualitativa las percepciones de los participantes en riesgo de exclusión, acerca de la radio universitaria, independientemente de que estuvieran o no en privación de su libertad. Para ello, de forma semanal, durante un periodo de dos años, se ha trabajado con estas personas en el diseño de un espacio radiofónico propio a partir del cual se ha ido modulando y controlando si ese deterioro de su identidad, fruto de un contexto social poco favorable, ha ido mejorando y reestructurándose gracias a la participación de este tipo de iniciativas.

Antes de iniciar ambas actividades, en estos dos escenarios desfavorecidos se partía de la premisa o hipótesis inicial de que la radio OndaCampus, en su condición de emisora universitaria, supone un instrumento de enorme utilidad como herramienta de reinserción social, formativa y terapéutica.

Para poder validar o refutar la hipótesis, una de las piedras angulares del método empleado ha sido la confección de un trabajo exploratorio basado en la experiencia real de trabajo con estas personas en riesgo de exclusión (realización de programas de radio semanales durante dos años consecutivos). Una vez puesta en marcha la iniciativa es cuando se ha optado por incluir la entrevista en nuestra metodología de análisis científico. El marcado carácter social y de reinserción de ambos proyectos requería la utilización de un recurso ágil y flexible que permitiera recabar aspectos concretos a partir de una serie de variables como las actitudes, creencias, valores, etc. Se ha entrevistado a un total de 18 participantes de la actividad ("Ventana de la Ciencia" y "E-Misión Color") entre coordinadores de proyecto; beneficiarios del proyecto; impulsadores del proyecto y responsables de servicios universitarios implicados en el desarrollo e impulso de los proyectos sociales. Concretamente, se han realizado las siguientes entrevistas (Tabla 1): 
Tabla 1

Perfiles entrevistas

\begin{tabular}{|l|l|}
\hline \multicolumn{2}{|c|}{ Entrevistas realizadas } \\
\hline La Ventana de la Ciencia & Internos: 3 \\
& Ex internos: 1 \\
& Unidad Cultura Científica Universidad: 1 \\
& Coordinación: 3 \\
& Jefe tratamiento: 1 \\
\hline E-Misión Color & Impulsor proyecto: 1 \\
& Monitores centro: 2 \\
& Usuarios/as centro: 4 \\
& Coordinación: 2 \\
\hline
\end{tabular}

Fuente: Elaboración propia.

Para poder analizar el tipo de respuesta, se han diseñado una entrevista semiestructurada, donde existían una serie de preguntas tipo pero con posibilidad, en todo momento, de ampliación de las respuestas por parte de los entrevistados. Concretamente, se han diseñado entrevistas con preguntas como las que se citan a continuación (Tabla 2):

Tabla 2

Cuestiones planteadas en base al perfil

\begin{tabular}{|c|c|}
\hline \multicolumn{2}{|c|}{ Preguntas planteadas } \\
\hline \multirow[t]{4}{*}{ Impulsadores de proyecto } & ¿Por qué decide dar impulso a este proyecto? \\
\hline & ¿Qué aporta su institución al proyecto radiofónico? \\
\hline & $\begin{array}{l}\text { ¿Por qué elige la radio universitaria como } \\
\text { coordinadora del proyecto? }\end{array}$ \\
\hline & Etc... \\
\hline \multirow[t]{4}{*}{ Beneficiarios del proyecto } & $\begin{array}{l}\text { ¿Cómo recibiste la notificación de participación en } \\
\text { el proyecto? }\end{array}$ \\
\hline & ¿Cómo afrontaste su desarrollo? \\
\hline & ¿Qué opinas de la metodología empleada? \\
\hline & Etc... \\
\hline \multirow[t]{3}{*}{ Coordinadores proyecto } & ¿Cómo surge la iniciativa de este programa? \\
\hline & $\begin{array}{l}\text { ¿Qué metodología se ha empleado para el } \\
\text { desarrollo del programa? }\end{array}$ \\
\hline & Etc... \\
\hline \multirow[t]{3}{*}{ Responsables de servicios universitarios } & $\begin{array}{l}\text { ¿Qué relación tiene su servicio con la radio } \\
\text { universitaria? }\end{array}$ \\
\hline & $\begin{array}{l}\text { ¿Qué participación tiene su servicio en el } \\
\text { proyecto? }\end{array}$ \\
\hline & Etc.. \\
\hline
\end{tabular}

Fuente: Elaboración propia. 
En cuanto a la realización de las entrevistas se realizaron de la siguiente forma:

- Beneficiarios de proyectos y coordinadores: entrevistas en persona.

- Responsables de servicios: entrevistas telefónica.

- Impulsores de proyectos: entrevistas por correo electrónico.

Somos conscientes que las entrevistas vía telefónica y vía mail no son la manera más adecuada de sacar conclusiones a este tipo de proyectos, ya que se pierde el contacto personal que es uno de los puntos fuertes de esta investigación. En nuestro caso, vemos subsanada esta distancia dado que en la mayoría de los casos, existe una relación profesional con las personas entrevistadas.

\section{RESULTADOS}

Las radios universitarias en España nacen con un carácter eminentemente formativo. A través de la oferta de prácticas adicionales a las académicas, las emisoras universitarias proporcionan a los alumnos y alumnas de los grados de Ciencias de la Información y la Comunicación, conocimientos prácticos a través del desarrollo de programas de radio de diferentes géneros y formatos.

La proliferación de este tipo de emisoras, propicia la evolución formal de las mismas, dotándolas de un papel importante y fundamental en la institución académica a la que pertenecen, la universidad. Casi de manera natural, nacen como una herramienta que refuerza el cumplimiento de la función social de la enseñanza superior. De tal forma, que sobrepasa los límites de la docencia adicional a la académica, para llegar a educar, en cierta medida, a la sociedad.

Analizando las entrevistas realizadas, 18 en concreto, lo que supone el $50 \%$ de la población del proyecto "E-Misión Color" y el $40 \%$ de la población del proyecto de "La Ventana de la Ciencia", podemos observar que se suceden durante el análisis una serie de palabras clave, a través de un análisis etiquetador morfosintáctico, que permiten dibujar tres grandes funciones sociales (figura 2) dentro de la radio universitaria que justifican su función como herramienta para la inclusión social. Estas palabras claves han sido obtenidas a partir de la transcripción de cada una de las entrevistas realizadas y la posterior extracción mediante las palabras más importantes del texto.

A partir de esta sucesión de palabras con peso o relevancia es posible establecer una serie de análisis tipológicos de funciones en la radio universitaria en lo concerniente a su papel como herramienta de reinserción social. 


\section{Funciones sociales naturales \\ Funciones sociales accidentales}

\section{Funciones sociales Transversales}

Figura 2. Funciones de la radio universitaria (tras análisis de resultados)

Fuente: Elaboración propia.

1) Funciones sociales naturales: en este análisis aparecen palabras claves como competencias y habilidades sociales, libertad de expresión, política y religiosa, alfabetización mediática, divulgación científica y/o cultural, orientación vocacional/laboral, radio universitaria y RSU. En cuanto a esta función y valores, tomamos como referencia la entrevista realizada a los monitores del Centro Social Gurugú, Abel y Víctor, quienes definían "E-Misión Color" como un proyecto social de defensa, de lucha de diferentes barrios. Destaca en las entrevistas el marcado carácter de responsabilidad que se otorga a la universidad, realizando declaraciones como por ejemplo que "La universidad no debe estar nunca desconectada de la ciudad ni de su funcionamiento, todo lo contrario." "Al haber tenido que hablar de científicos, me ha aportado mucha cultura y mucho saber de la ciencia y yo me he sorprendido a mí mismo el interés que tengo sobre la ciencia. Empezar hablando de Galileo Galilei, de Ramón y Cajal, que yo pensaba que Ramón y Cajal eran dos personas y ahora resulta que era solo una (risas)".

2) Funciones sociales accidentales: aparecen de manera repetida conceptos como competencia y habilidades audiovisuales, competencias comerciales, educación ambiental, educación formal, educación para la paz, tolerancia e igualdad, interculturalidad e inserción social. En este punto, recurrimos a la información recogida de los diferentes beneficiarios de algunos proyectos sociales de OndaCampus. Como son los casos de Marta y Pauli "E- Misión Color" en primer lugar donde hablan del enorme aprendizaje que supone la radio universitaria: "Aprendo cosas nuevas, me lo paso bien. He notado mejoría a la hora de hablar y en lectura no mucho, pero bueno también sí. Antes escribía palabras mal y ahora las escribo y las digo bien". Impresiones que se suman a las realizadas por los internos del Centro Penitenciario de Badajoz, en referencia a la inserción social, y a esa inclusión que es objeto de esta investigación que aquí se expone "...la gente, lo que estamos haciendo nosotros, el día que salgamos a la calle, que no diga "ha estado en la cárcel se ha vuelto un criminal". Que diga "ha estado en la cárcel y le ha servido de algo", ha crecido, ha 
aprendido cosas nuevas, ha cambiado como persona, es una persona mejor, ya no es el mismo de antes".

3) Funciones sociales transversales: democracia, ética, igualdad, respeto, responsabilidad, solidaridad y tolerancia. En relación a este tipo de funciones sociales, podemos comprobar que todas se corresponden con los valores de ciudadanía, que ayudan al desarrollo personal de la sociedad y que son asimilados, inconscientemente, en los programas por oyentes y productores de los mismos. En lo referente a estos valores, que son más difíciles de detectar, hemos comprobado su cumplimiento a través de la observación de las actitudes de los entrevistados. Su sentimiento de gratitud y el seguimiento de su evolución a lo largo de proyectos en los que hemos estado implicados de manera laboral a través de los mismos. "Es complicado comunicar con palabras que un programa de radio aporta valores de democracia, amplia la actitud ética o desarrolla el respeto y la responsabilidad". "He aprendido a trabajar en equipo, aquí me siento más responsable y con la obligación de dar una respuesta a la sociedad, pues la espera de mí." Son valores abstractos que se van detectando a través del contacto continuo y el desarrollo en este tipo de iniciativas.

Las declaraciones del $95 \%$ de los entrevistados corroboran la función social natural de la radio universitaria a través de la ejecución de sus proyectos sociales. La radio universitaria, pues, se consolida evolutivamente como una herramienta para el cumplimiento de la función social. A través de nuestro análisis, hemos prestado especial atención a aquellos proyectos sociales que más determinan la función social en OndaCampus, fortaleciendo la consecución de la función social y, en concreto, la inclusión de personas en riesgo de exclusión.

\section{CONCLUSIONES}

A lo largo de nuestra investigación, podemos concluir la consecución de nuestro objetivo así como la validación de nuestra hipótesis, ya planteada en el apartado 1 de introducción, por lo siguiente:

- La radio universitaria puede consolidarse como una herramienta eficaz para transportar fielmente la función social debido a que se ha convertido en un medio de comunicación libre, que permite a la institución académica el trasvase de conocimientos a toda la sociedad, en general. Este hecho le permite establecer un puente cada vez más sólido que rompe con las fronteras históricamente establecidas y flanqueadas por las cuatro paredes que conforman el campus universitario.

- Concluimos que la metodología llevada a cabo es la idónea por encontrarse adaptada al tipo de colectivo al que vayan dirigidas las acciones radiofónicas. Esto implica un proceso de contacto continuo entre radio universitaria y 
sociedad que propicia la relación entre ambos, aumentando de tal forma, la RSU. Además, determinamos, que las emisoras universitarias transmiten tres tipos de funciones sociales a través de sus valores: función social natural, función natural accidental y función social transversal. Funciones que ayudan al individuo a la conformación y asimilación de conocimientos y aptitudes que le faciliten sus relaciones sociales y profesionales.

A modo de conclusión final, podemos afirmar que las emisoras universitarias y, en concreto la nuestra emisora, objeto de estudio, OndaCampus, pueden constituirse como una herramienta eficaz para desempeñar la función social, cuyo objetivo y desempeño principal es tender un puente, sonoro, entre la Universidad y la Sociedad y, por extensión, ayudar a la inclusión de personas en riesgo de exclusión social por diferentes circunstancias.

\section{Referencias}

Aguaded, J.I. \& Contreras, P. (2011). La radio universitaria como servicio público para una ciudadanía democrática. La Coruña: NETBIBLO.

Aguaded, J. \& Martín-Pena, D. (2013). Educomunicación y radios universitarias: panorama internacional y perspectivas futuras. Revista Latinoamericana de Comunicación Chasqui. (124), 63-70.

Antequera, J. \& Obregón, R. (2002). La radio como dinamizadora de procesos sociales y culturales en Barranquilla (Colombia). Investigación y Desarrollo. 10 (2), 146-169.

Bueno Campos, E. (2017). La Tercera Misión de la Universidad: el reto de la transferencia del conocimiento, Revista de Investigación en Gestión de la Innovación y Tecnología, 41, marzo-abril, 1-8.

Blumler, J. \& Katz, E. (Eds.) (1974). The Uses of Mass Communications. Currents Perspectives on Gratifications Research. Beverly Hills: Editorial Sage,

Bretones, M.T. (1997). Funciones y efectos de los medios de comunicación de masas: modelos de análisis. En: M.T. Bretones, La comunicación política mediática y sus dimensiones, 137-196.

Casajús, L. (2011). Radio universitaria en América Latina: escenarios y perspectivas. En J. Aguaded, \& P. Contreras, La radio universitaria como servicio público para una ciudadanía democrática (pp. 70-81). La Coruña: NETBIBLO.

Casas, M. (2005). Nueva universidad ante la sociedad del conocimiento. Revista de Universidad y Sociedad del Conocimiento, 2 (2), 1-17.

Chomsky, N. y Hernan, E. (1990). Los guardianes de la libertad. Barcelona: Grijalbo. 
Contreras, P. (2012). La voz de los presos en la radio universitaria: puentes con la ciudadanía. La experiencia de UniRadio, Universidad de Huelva. En C. Espino \& D. Martín-Pena, Las radios universitarias, más allá de la radio. Las TIC como recursos de interacción radiofónica (pp. 153-165). Barcelona: UOC.

Contreras, P. González-Mairena, M. \& Aguaded, I. (2014). Programar una radio social en la universidad: el Propósito Penélope de UniRadio. EDMETIC. Revista de Educación Mediática y TIC, 3 (1), 112-130.

Ferrer, J. (2014). La función social de la Universidad, El País, Tribuna, 26 de diciembre..

Garcés, Raúl (2007). "La crisis de los discursos radiofónicos". En Revista Latina de Comunicación Social, núm. 62. Recuperado el 20 de noviembre de2019: http://www.ull.es/publicaciones/latina/200719RaulGarces.htm.

González Conde, J. (2003): “Ámbitos de actuación de la radio educativa y su integración en el contexto escolar". En Red Digital. Revista de Tecnología de la información y comunicación educativas, núm. 4. Centro Nacional de Información y Comunicación Educativa (CNICE): Madrid. Recuperado el 20 de noviembre de 2019 de: http://reddigital.cnice.mecd.es/4/firmas/julia_ind.html

González-Mairena, M. \& Ramos, A. (2012). La audiencia en los debates radiofónicos a través de Facebook. La experiencia de UniRadio, Universidad de Huelva. En C. Espino \& D. Martín-Pena, Las radios universitarias, más allá de la radio. Las TIC como recursos de interacción radiofónica. (pp. 229-243). Barcelona: UOC.

Lazarsfeld, P. \& Merton, R. (1985). Comunicación de masas, gustos populares y acción social organizada. En M. Moragás, Sociología de la comunicación de masas. Barcelona: Gustavo Gili.

López, M. (2005). La construcción de la identidad a través de la radio digital universitaria. En: Marín, A. (Presidencia), II Congreso Iberoamericano de Comunicación Universitaria, Granada, España.

López Vidales, N. (2011): “La radio se transforma: Nuevas tecnologías, nuevos hábitos de consumo y nuevos perfiles para el medio más cercano". En Radio 3.0 Una nueva radio para una nueva era. Madrid: Fragua, pp. 15-40.

Marta-Lazo, C. y Segura-Anaya, A. (2011). La radio educativa en la era del dial infinito. (pp. 341 -255). En M. Gértrudix Barrio, F. Gértrudix Barrio y F. García García (coords.). Actas II Congreso Internacional Sociedad Digital: espacios para la interactividad y la inmersión.

Martín-Pena, D. \& Aguaded, I. (2016). La radio universitaria en España: comunicación alternativa de servicio público para la formación. Comunicación y sociedad, (25), 237265.

Martín-Pena, D. \& Espino, C. (2014). Contenido, estructura y función social en la programación de las radios universitarias. EDMETIC. Revista de Educación Mediática y TIC. 3 (1), 2943. 
Martín-Pena, D. (2014) Las radios universitarias en España: plataformas interactivas y redes de colaboración. Tesis doctoral no publicada. Universidad de Huelva.

Martín-Pena, D., Parejo, M. \& Vivas, A. (2016). La radio universitaria: Gestión de la información, análisis y modelos de organización. Barcelona, Gedisa.

Merayo, A. (2000). Identidad, sentido y uso de la radio educativa. En: III Congreso Internacional Cultura y Medios de Comunicación. Salamanca: Ediciones Universidad Pontificia de Salamanca.

Mora, B. (2016). La radio universitaria como servicio público a la ciudadanía: el caso de Uniradio-UHU. Tesis doctoral publicada. Universidad de Huelva.

Muñoz Alonso, A., Monzón, C., Rospir, J.I. \& Dader, J.L. (1990). Opinión pública y comunicación política. Madrid: Eudema.

Parejo, M., Vivas, A. \& Martín-Pena, D. (2018). Divulgación científica y función social en las universidades. Madrid: Editorial Pirámide.

Pinto-Zúñiga, R. \& Martín-Pena, D. (2017). La responsabilidad social a través de las ondas universitarias españolas. Anuario Electrónico de Estudios en Comunicación Social "Disertaciones". 10 (1), 158-176.

Rotman, A. (2014). Las radios universitarias argentinas frente a un nuevo paradigma de la comunicación. En D. Martín-Pena \& M.A. Ortiz, Radios universitarias en América y Europa (pp.39-46). Madrid: Fragua.

Sols, J. (2016). El pensamiento de Ignacio Ellacuría acerca de la función social de la Universidad. Arbor. Ciencia, pensamiento y cultura, vol. 192-782, noviembrediciembre.

Valleys, F. (2014). ¿Qué es la responsabilidad social universitaria? [consulta: 23 de abril de 2018]. Disponible en: http://palestra.pucp.edu.pe/index.php?id=111.

Wolf, M. (1991). La investigación de la comunicación de masas. Crítica y perspectivas. Barcelona: Editorial Paidós, 22. 